\title{
TOPOLOGICAL DYNAMICS ON NILMANIFOLDS
}

L. AUSLANDER, ${ }^{1}$ F. HAHN AND L. MARKUS ${ }^{2}$

Communicated by W. S. Massey, January 18, 1961

There has always been a lack of examples of compact manifolds which are minimal sets under the action of the real line, minimal meaning that each orbit is dense in the manifold. All tori admit such an action and G. A. Hedlund [4] has given examples of 3-manifolds having such an action.

The action of a group $T$ on a metric space $X$ is said to be distal if for any two distinct points $x, y \in X$ the distance between $t x$ and $t y$ bounded away from zero for $t \in T$. In the works of R. Ellis [1] and W. H. Gottschalk [2] the question of whether a distal minimal set is equicontinuous has arisen. Indeed, R. Ellis has shown that if $X$ is locally compact and zero dimensional and if $T$ acts on $X$ so the action is distal and minimal then the action is equicontinuous.

The authors have shown that every compact nilmanifold $M$ admits a flow under which $M$ is minimal. This action is even real analytic. Thus there is no scarcity of manifolds which are minimal under a flow. We have also shown that these actions are distal. If these actions were equicontinuous it would follow that $M$ would be a torus [3]. Since there are nilmanifolds which are not tori, we have shown the existence of analytic flows on compact manifolds which are distal, minimal, but not equicontinuous.

Our basic approach is as follows:

Lemma. If $W$ is an open subset of a connected, simply connected, nilpotent Lie group $N$ with discrete uniform subgroup $D$, then the set swept out by the one parameter subgroups containing some element of $W$ contains a fundamental domain for $N / D$.

THEOREM. If $D$ is a discrete uniform subgroup of a connected, simply connected, nilpotent Lie group $N$ then the set of points of $N$, which iie on one parameter subgroups whose projection on $N / D$ is dense, is a set of category II.

By a relatively straightforward calculation we can show that the action of any one parameter subgroup on $N / D$ is distal. From Ellis

\footnotetext{
${ }^{1}$ Research supported by N. S. F. Grant 15565 and O. O. R. contract SAR-DA19-020 ORD-5254.

2 Research supported by N. S. F. Grant 11287.
} 
[1] we can conclude that the action of each one parameter subgroup is pointwise almost periodic. The theorem then shows that there exist one parameter subgroups such that $N / D$ is an orbit closure. Since the action is pointwise almost periodic it follows that $N / D$ is minimal [3].

Complete proofs of these results will be published elsewhere.

\title{
REFERENCES
}

1. R. Ellis, Distal transformation groups, Pacific J. Math., vol. 8 (1958) pp. 401405.

2. W. H. Gottschalk, Minimal sets: An introduction to topological dynamics, Bull. Amer. Math. Soc. vol. 64 (1958) pp. 336-351.

3. W. H. Gottschalk and G. A. Hedlund, Topological dynamics, Amer. Math. Soc. Colloquium Publications, vol. 36, 1955.

4. G. A. Hedlund, Fuchsian groups and transitive horocycles, Duke J. Math. vol. 2 (1936) pp. 530-542.

\author{
INDIANA UNIVERSITY, \\ YALE UNIVERSITY AND \\ UNIVERSITY OF MINNESOTA
}

